Provided for non-commercial research and education use. Not for reproduction, distribution or commercial use.

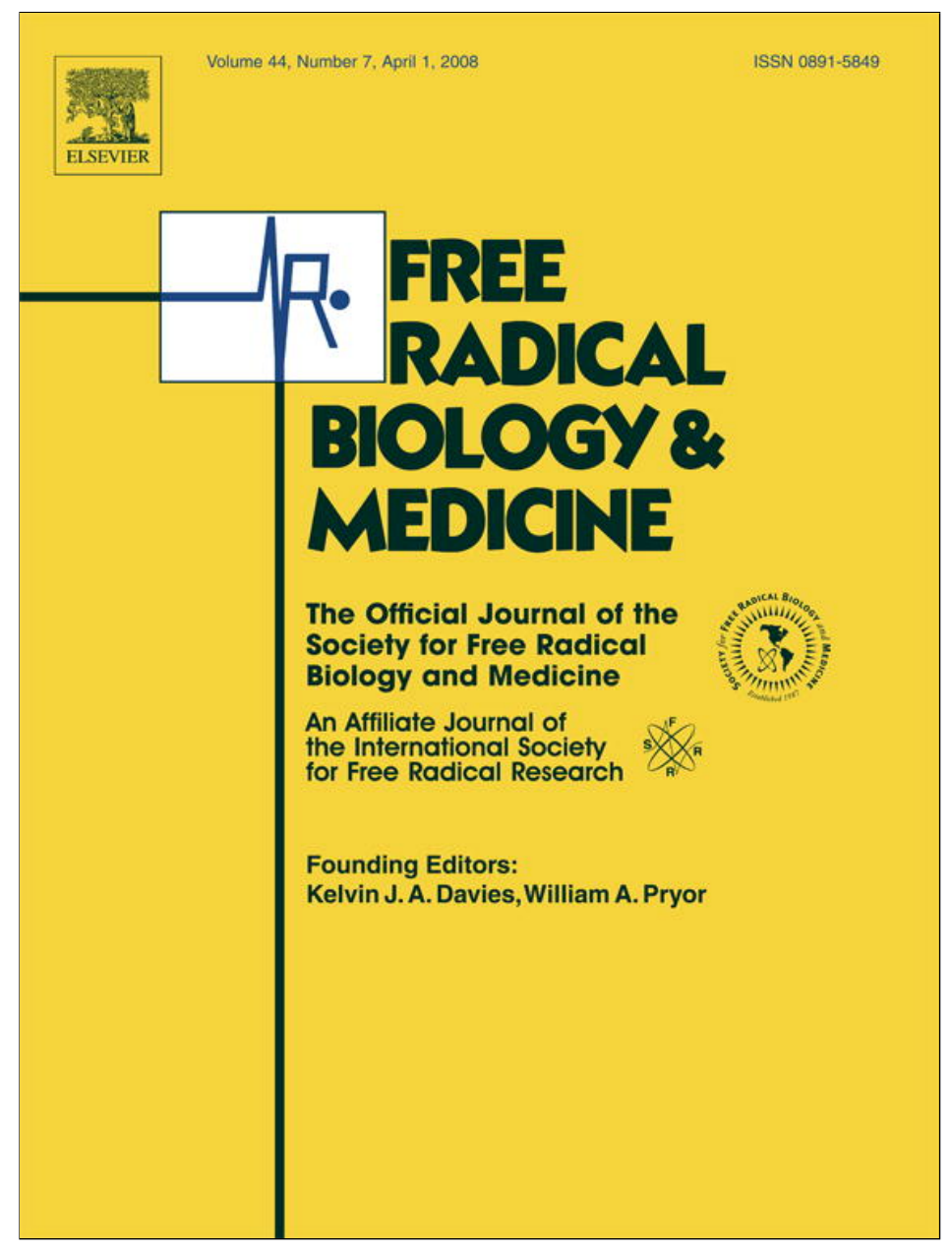

This article appeared in a journal published by Elsevier. The attached copy is furnished to the author for internal non-commercial research and education use, including for instruction at the authors institution and sharing with colleagues.

Other uses, including reproduction and distribution, or selling or licensing copies, or posting to personal, institutional or third party websites are prohibited.

In most cases authors are permitted to post their version of the article (e.g. in Word or Tex form) to their personal website or institutional repository. Authors requiring further information regarding Elsevier's archiving and manuscript policies are encouraged to visit:

http://www.elsevier.com/copyright 


\title{
Role of mitochondria and reactive oxygen species in dendritic cell differentiation and functions
}

\author{
Annalisa Del Prete ${ }^{\mathrm{a}, \mathrm{b}, 1}$, Patrizia Zaccagnino ${ }^{\mathrm{a}, 1}$, Marco Di Paola ${ }^{\mathrm{c}, 1}$, Maddalena Saltarella ${ }^{\mathrm{a}}$, \\ Constanza Oliveros Celis ${ }^{\mathrm{a}}$, Beatrice Nico ${ }^{\mathrm{d}}$, Giuseppe Santoro ${ }^{\mathrm{e}}$, Michele Lorusso ${ }^{\mathrm{a}, \mathrm{c}, *}$ \\ ${ }^{a}$ Department of Medical Biochemistry, Biology, and Physics, University of Bari, 70124 Bari, Italy \\ ${ }^{\mathrm{b}}$ Istituto Clinico Humanitas, Rozzano, Milano, Italy \\ ${ }^{\mathrm{c}}$ Institute of Biomembrane and Bioenergetics, CNR, Bari, Italy \\ d Department of Human Anatomy and Histology, University of Bari, 70124 Bari, Italy \\ e Department of Internal Medicine and Human Oncology, University of Bari, 70124 Bari, Italy
}

Received 12 October 2007; revised 21 December 2007; accepted 22 December 2007

Available online 11 January 2008

\begin{abstract}
Dendritic cells (DC) are potent antigen-presenting cells capable of inducing T and B responses and immune tolerance. We have characterized some aspects of energy metabolism accompanying the differentiation process of human monocytes into DC. Compared to precursor monocytes, DC exhibited a much larger number of mitochondria and consistently (i) a higher endogenous respiratory activity and (ii) a more than sixfold increase in ATP content and an even larger increase in the activity of the mitochondrial marker enzyme citrate synthase. The presence in the culture medium of rotenone, an inhibitor of the respiratory chain Complex I, prevented the increase in mitochondrial number and ATP level, without affecting cell viability. Rotenone inhibited DC differentiation, as revealed by the observation that the expression of CD1a, which is a specific surface marker of DC differentiation, was strongly reduced. Cells cultured in the presence of rotenone displayed a lower content of growth factor-induced, mitochondrially generated, hydrogen peroxide. A similar drop in ROS was observed upon addition of catalase, which caused functional effects similar to those produced by rotenone treatment. These results suggest that ROS play a crucial role in DC differentiation and that mitochondria are an important source of ROS in this process.
\end{abstract}

(C) 2008 Elsevier Inc. All rights reserved.

Keywords: Dendritic cells; Mitochondria; Reactive oxygen species; Oxidative phosphorylation; Free radicals

Effective immune responses require correct localization and functioning of dendritic cells (DC). Dendritic cells are the most potent and versatile antigen-presenting cells, with a unique ability to induce specific immune responses as well as tolerance $[1,2]$. In peripheral tissues they reside in an immature state

Abbreviations: DC, dendritic cells; ROS, reactive oxygen species; GM-CSF, granulocyte-macrophage colony-stimulating factor; IL-4, interleukin-4; CCCP, carbonyl cyanide 3-chlorophenylhydrazone; DTNB, 5,5'-dithiobis-(2-nitrobenzoic acid); DCFH-DA, 2' 7' $^{\prime}$-dichlorodihydrofluorescein diacetate; $\mathrm{LDH}$, lactate dehydrogenase.

* Corresponding author. Department of Medical Biochemistry, Biology, and Physics, University of Bari, 70124 Bari, Italy. Fax: +39 0805478109.

E-mail address: m.lorusso@biochem.uniba.it (M. Lorusso).

1 These authors contributed equally to this work. waiting for incoming antigens. After capturing and processing the antigens, DC undergo a maturation process which culminates in dramatic changes in functions and migratory properties $[3,4]$. The localization of mature DC to the draining lymph nodes coincides with the presentation of processed antigens to naïve $\mathrm{T}$ cells, triggering the initiation of specific immune responses $[2,5]$. An in vitro method to differentiate immature DC from $\mathrm{CD} 14^{+}$monocyte precursors cultured in the presence of granulocyte-macrophage colony-stimulating factor (GM-CSF) and interleukin-4 (IL-4) is very well established [6,7].

Reactive oxygen species (ROS) have been identified as important second messengers involved in the transduction of several signaling pathways $[8,9]$, gene expression, and cell proliferation [10]. Furthermore, recent studies have shown that growth factors, through the actions of their specific receptors, are able to increase 
the relative levels of intracellular ROS [11,12]. However, the mechanism involved and the origin of intracellular ROS are not well understood [13].

The main source of ROS during normal metabolism is represented by the mitochondrial electron-transport chain activity [14]. In particular, Complex I and Complex III have been identified as the major sites of ROS formation $[15,16]$. An alternative site of ROS production is the NADPH oxidase multicomponent enzyme system, which is very well characterized in phagocytic cells (neutrophils and macrophages) as an essential mechanism of defense against pathogens [17-19]. Recent studies showed that, whereas NADPH oxidase activation is required for DC killing of intracellular Escherichia coli, no role in DC differentiation or functions can be ascribed to NADPH oxidasederived oxygen radicals [20,21]. However, an involvement of ROS has been recently described in the activation and maturation process of DC [22-24].

The present study was aimed at characterizing the differentiation from monocyte precursors into immature DC, in terms of biochemical features as well as phenotypical and functional aspects. In particular, we addressed the question whether ROS are involved in the differentiation process. Based on the effects elicited by the respiratory chain Complex I inhibitor rotenone on the morphological as well as biochemical alterations accompanying the differentiation, we suggest that the increased levels of ROS, which seem to trigger the process, depend on the mitochondrial respiratory activity.

\section{Materials and methods}

\section{Cell culture media and reagents}

rhGM-CSF and rhIL-4 were from R\&D Systems (Minneapolis, MN, USA). Cytokines were endotoxin free as assessed by the Limulus amebocyte assay (BioWhittaker, Walkersville, MD, USA). FITC-dextran, catalase, rotenone, oligomycin, carbonyl cyanide 3-chlorophenylhydrazone (CCCP), protease inhibitor cocktail, 5,5'-dithiobis-(2-nitrobenzoic acid) (DTNB), bovine heart lactate dehydrogenase (LDH), $\mathrm{NAD}^{+}$, and the bioluminescence somatic cell ATP assay kit were purchased from Sigma (St. Louis, MO, USA). The annexin V-FITC/propidium iodide apoptosis detection kit was obtained from BD Bioscience (Mountain View, CA, USA). 2',7'-Dichlorodihydrofluorescein diacetate (DCFH-DA) and MitoTracker red were from InvitrogenMolecular Probes (Eugene, OR, USA).

\section{Dendritic cell culture generation and characterization}

Peripheral blood mononuclear cells were isolated by standard Ficoll-Paque (Amersham, Buckinghamshire, UK) gradient centrifugation from buffy coats (obtained through the courtesy of the local blood bank, Polyclinic Hospital, Bari, Italy). Monocytes were purified by immunomagnetic separation using anti-CD14conjugated magnetic microbeads (Miltenyi, Bergisch Gladbach, Germany) and cultured for 6 days at $1 \times 10^{6} / \mathrm{ml}$ in RPMI 1640 medium (Biochrom AG, Berlin, Germany) supplemented with $10 \%$ heat-inactivated FBS (Euro Clone, Milan, Italy), GM-CSF
(50 ng/ml), and IL-4 (20 ng/ml). DC maturation was induced by the addition of lipopolysaccharide (LPS) at the final concentration of $100 \mathrm{ng} / \mathrm{ml}$. Cells were cultured for a further $48 \mathrm{~h}$.

In rotenone experiments, $50 \mathrm{nM}$ rotenone was added to the culture medium $10 \mathrm{~min}$ after GM-CSF and IL-4. After a further 10-min incubation, the cell suspension was supplemented with $10 \%$ FBS and cultured for 6 days. In catalase experiments monocytes were incubated $15 \mathrm{~min}$ with $500 \mathrm{IU} / \mathrm{ml}$ catalase before the addition of GM-CSF, IL-4, and FBS. Respiratory activity in monocytes was inhibited by around $75 \%$ by rotenone, whereas it was unaffected by catalase.

Surface phenotype analysis was performed using anti-CD14 (Miltenyi), anti-CD1a (Serotec, Oxford, UK), anti-CD83, and anti-CCR5 monoclonal antibodies (R\&D Systems). Mannose receptor-mediated endocytosis was analyzed by FITC-dextran uptake at $37^{\circ} \mathrm{C}$. Cells were analyzed with a FACScan flow cytometer (BD Bioscience) using CellQuest software.

\section{Electron microscopy}

Monocytes and DC were fixed for $2 \mathrm{~h}$ in $0.1 \mathrm{M}$ phosphate buffer containing 3\% glutaraldehyde, washed in the same buffer, and then postfixed in $1 \% \mathrm{OsO}_{4}$ at $4{ }^{\circ} \mathrm{C}$. Afterward, cells were scraped with a rubber bar, dehydrated in graded ethanol, and embedded in Epon 812. Sixty-nanometer ultrathin sections were cut with a diamond knife on a LKB-V Ultratome, stained with uranyl acetate followed by lead citrate, and examined under a Zeiss EM 109 electron microscope. For the morphometrical analysis, mitochondria of 50 cells for each sample were counted on the electron micrographs at a final magnification of $12,000 \times$ by computer-aided analysis.

The mean value in each micrograph, the final mean value for all the micrographs, and the standard error (SEM) were calculated.

\section{Measurement of oxygen consumption rate}

Cellular respiratory activity was measured polarographically with a Clark-type electrode (Yellow Spring Instruments, Yellow Spring, OH, USA), in an all-glass reaction chamber, magnetically stirred, at $37^{\circ} \mathrm{C}$. Monocytes $\left(4 \times 10^{6}\right)$ or DC $\left(2 \times 10^{6}\right)$ were suspended in $0.5 \mathrm{ml}$ of PBS containing $5 \mathrm{mM}$ glutamine, $5 \mathrm{mM}$ pyruvate, and $2.5 \mathrm{mM}$ malate. State 4 and uncoupled respiration were obtained by adding $2 \mu \mathrm{g} / \mathrm{ml}$ oligomycin and $0.5 \mu \mathrm{M}$ CCCP.

\section{Determination of citrate synthase activity}

Citrate synthase activity was measured spectrophotometrically by following the reduction of DTNB at $412-360 \mathrm{~nm}$ $\left(\varepsilon=13.6 \mathrm{mM}^{-1} \times \mathrm{cm}^{-1}\right)$. Cells $\left(1 \times 10^{6}\right)$ were pelleted and resuspended in $0.2 \mathrm{ml}$ of a medium containing $25 \mathrm{mM}$ phosphate buffer ( $\mathrm{pH} 7.4$ ), $5 \mathrm{mM} \mathrm{MgCl}_{2}$, and $1 \mu$ of protease inhibitor cocktail. After freezing and thawing three times, aliquots (20 to $120 \mu \mathrm{l})$ of the suspension were added to a reaction mixture consisting of $10 \mathrm{mM}$ Tris-Cl buffer ( $\mathrm{pH} 7.4), 0.3 \mathrm{mM}$ acetylcoenzyme A, $0.2 \mathrm{mM}$ DTNB, and $0.2 \%(\mathrm{v} / \mathrm{v})$ Triton X-100. The reaction was started by the addition of $0.5 \mathrm{mM}$ oxaloacetate. 


\section{Measurement of intracellular ATP content}

ATP concentration was determined using a bioluminescence somatic cell assay kit, following the manufacturer's instructions. We used $2 \times 10^{4}$ cells of each sample. Chemiluminescence signals were acquired with a Perkin-Elmer Wallac 1420 VICTOR3 multilabel plate reader.

\section{Measurement of lactate production}

Analysis of lactate released into the culture medium was carried out spectrophotometrically by following $\mathrm{NAD}^{+}$reduction at $340 \mathrm{~nm}\left(\varepsilon=6.22 \mathrm{mM}^{-1} \times \mathrm{cm}^{-1}\right)$, essentially as described in [25]. Briefly, $100 \mu \mathrm{l}$ of cell suspension was withdrawn from culture plates and centrifuged. Cell-free supernatants were added to $900 \mu \mathrm{l}$ of a medium containing $467 \mathrm{mM}$ glycine, $280 \mathrm{mM}$ hydrazine sulfate, $2.6 \mathrm{mM}$ EDTA (pH 9.5), and $1 \mathrm{mM} \mathrm{NAD}^{+}$and the reaction was started by adding $10 \mathrm{IU}$ of $\mathrm{LDH}$.

\section{Fluorimetric determination of intracellular ROS}

Intracellular ROS levels were determined by measuring fluorescence intensity (excitation at $475 \mathrm{~nm}$, emission at $525 \mathrm{~nm}$ ) in cells suspended in serum-free medium and loaded with the redox-sensitive dye DCFH-DA [26]. The nonfluorescent DCFHDA readily diffuses into the cells, where it is hydrolyzed to the polar derivative $\mathrm{DCFH}$, which is in turn oxidized in the presence of $\mathrm{H}_{2} \mathrm{O}_{2}$ to the highly fluorescent DCF. Cells $\left(1 \times 10^{6} / \mathrm{ml}\right)$ were incubated with $5 \mu \mathrm{M}$ DCFH-DA in the dark at $37^{\circ} \mathrm{C}$. After $30 \mathrm{~min}$ incubation, cells were thoroughly washed quickly with a pulse spin and immediately suspended in $1 \mathrm{ml}$ of PBS. The ROSdependent oxidation of DCFH-DA to the fluorescent derivative was measured with a Jasco FP6200 spectrofluorimeter.

\section{Laser scanning confocal microscopy}

Monocytes $\left(1 \times 10^{6} / \mathrm{ml}\right)$ were incubated with $200 \mathrm{nM}$ MitoTracker red and $5 \mu \mathrm{M}$ DCFH-DA, for $30 \mathrm{~min}$ at $37^{\circ} \mathrm{C}$ in the dark. At the end of incubation, cells were washed two times with cold PBS and transferred onto a glass microscope slide. Confocal microscopy was performed with a Nikon Eclipse E600 microscope (Nikon Corp., Tokyo, Japan), equipped with argon-ion and helium-neon lasers, emitting at 488- and 543-nm wavelengths. Fluorescent signals emitted by DCF and MitoTracker red were quantified with the Nikon EZ C1 software (Nikon Corp., version 2.10, Coord Automatisering), which allows the quantitation of the fluorescence intensity profile of the pixels within a defined area including a single cell. Fluorescence intensities of the individual cells in both emission channels were corrected for the background by repeating the procedure in a cell-free field.

\section{Statistical analysis}

Results are expressed as means \pm SEM. Statistical significance was determined using Student's $t$ test. Differences were considered significant when $p<0.05$.

\section{Results}

Characterization of the mitochondrial oxidative phosphorylation system in monocytes and DC

The results of experiments aimed at characterizing the mitochondrial oxidative phosphorylation system in monocytes and DC are summarized in Fig. 1. The respirometric analysis shows that the differentiation of monocytes into DC was accompanied by a more than fivefold increase in endogenous respiratory activity (Fig. 1A). This activity was substantially inhibited by the ATP synthase inhibitor oligomycin and only slightly stimulated by the subsequent addition of the protonophore uncoupler CCCP, indicating that mitochondria were in an active (phosphorylating) state. The increase in respiratory control ratio (uncouplerstimulated vs oligomycin-inhibited respiration) observed in DC with respect to monocytes indicates an improvement in mitochondrial coupling during the differentiation process. This finding is substantiated by a significant increase in intracellular ATP content (Fig. 1B, open circles), which is in turn paralleled by the increase in the activity of the matrix marker enzyme citrate synthase (Fig. 1B, closed circles). LPS-induced maturation of DC, which was characterized by up regulation (around $90 \%$ positive cells) of CD83 and down regulation of CCR5 expression, did not
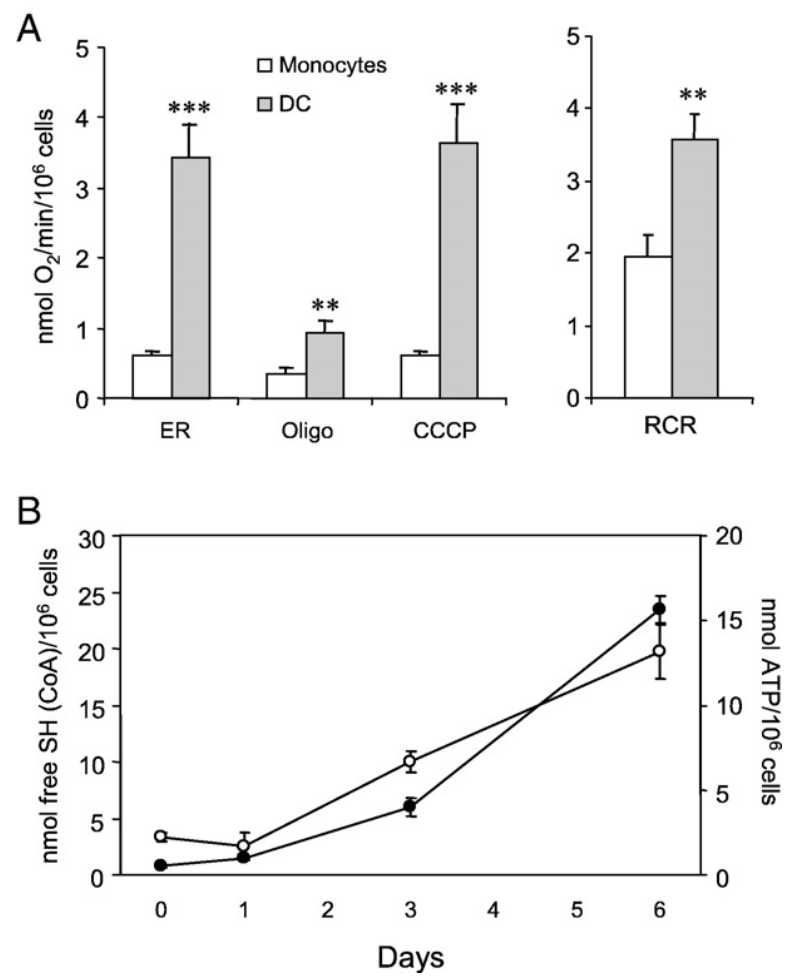

Fig. 1. Mitochondrial enzymatic activities in monocytes and monocyte-derived DC. (A) Cellular respiratory rate was measured in monocytes and in monocytederived DC cultured for 6 days as described under Materials and methods. ER, endogenous respiration. Where indicated oligomycin (Oligo, $2 \mu \mathrm{g} / \mathrm{ml}$ ) and CCCP $(0.5 \mu \mathrm{M})$ were added. RCR, respiratory control ratio as uncoupled vs oligomycininhibited respiratory rate $\left(* * p<0.01\right.$ and ${ }^{* * *} p<0.001 \mathrm{DC}$ vs monocytes). (B) Citrate synthase activity (closed circles) and intracellular ATP content (open circles) during the differentiation process from monocytes into DC. Values are reported as means \pm SEM from at least three different experiments. 
cause any significant variation in either the endogenous respiratory activity or the respiratory control ratio. Citrate synthase activity was also measured in mature DC and found to be almost unchanged (not shown).

\section{Ultrastructural features of monocytes and DC}

Ultrastructurally, monocytes appeared as rounded cells with a few organelles and a high nuclear-to-cytoplasmic ratio. They showed small mitochondria characterized by a large intermembrane space, irregularly swollen cristae, and electron-dense matrix ("condensed" mitochondria). Moreover, "orthodox" mitochondria, having a thin intermembrane space, slender and tubular cristae, and electron-transparent matrix, were also present (Fig. 2A). DC appeared as flat cells with abundant cytoplasm containing an irregularly sized nucleus, characterized by plasma membranes with numerous fillipodes and pseudopodes. Moreover, DC displayed a significant increment in the number of condensed mitochondria compared with monocytes (Fig. 2B).

The presence of rotenone in the culture medium gave rise to cells exhibiting a significant reduction in the number of mitochondria compared with control DC. Furthermore, mitochondria showed an electron-lucent matrix with evident lamellar cristae and an easily recognizable orthodox conformation (Fig. 2C).
Lipid droplets and profiles of rough endoplasmic reticulum were also present. The statistical analysis of these data is reported in Fig. 2D. The cell viability tested by propidium iodide/annexin $\mathrm{V}$ double staining was never below $90 \%$ either in control or in rotenone-treated cells (not shown).

\section{Phenotypical and functional characterization}

For monitoring DC differentiation we examined the surface density of CD14, a classical marker of monocytes, and of CD1a, which is specific for DC. The time course of CD14 and CD1a expression during the differentiation process is shown in Fig. 3. In control DC CD14 expression significantly started to decline at day +2 , tending to disappear over the 6-day culture period, whereas in rotenone-treated DC around 30\% positive cells were still detected at the end of the culture period (Fig. 3A). Conversely, rotenone-treated DC showed very low levels of CD1a over the time investigated, whereas control DC reached a very high level of expression of this marker at day +6 of the culture $(\approx 80 \%)$ (Fig. 3B). A statistical analysis of five independent experiments is reported in Fig. 3C. Chemokine receptor characterization of rotenone-treated cells showed that CCR5 expression was severely reduced (around 70\%) compared to control DC (not shown).
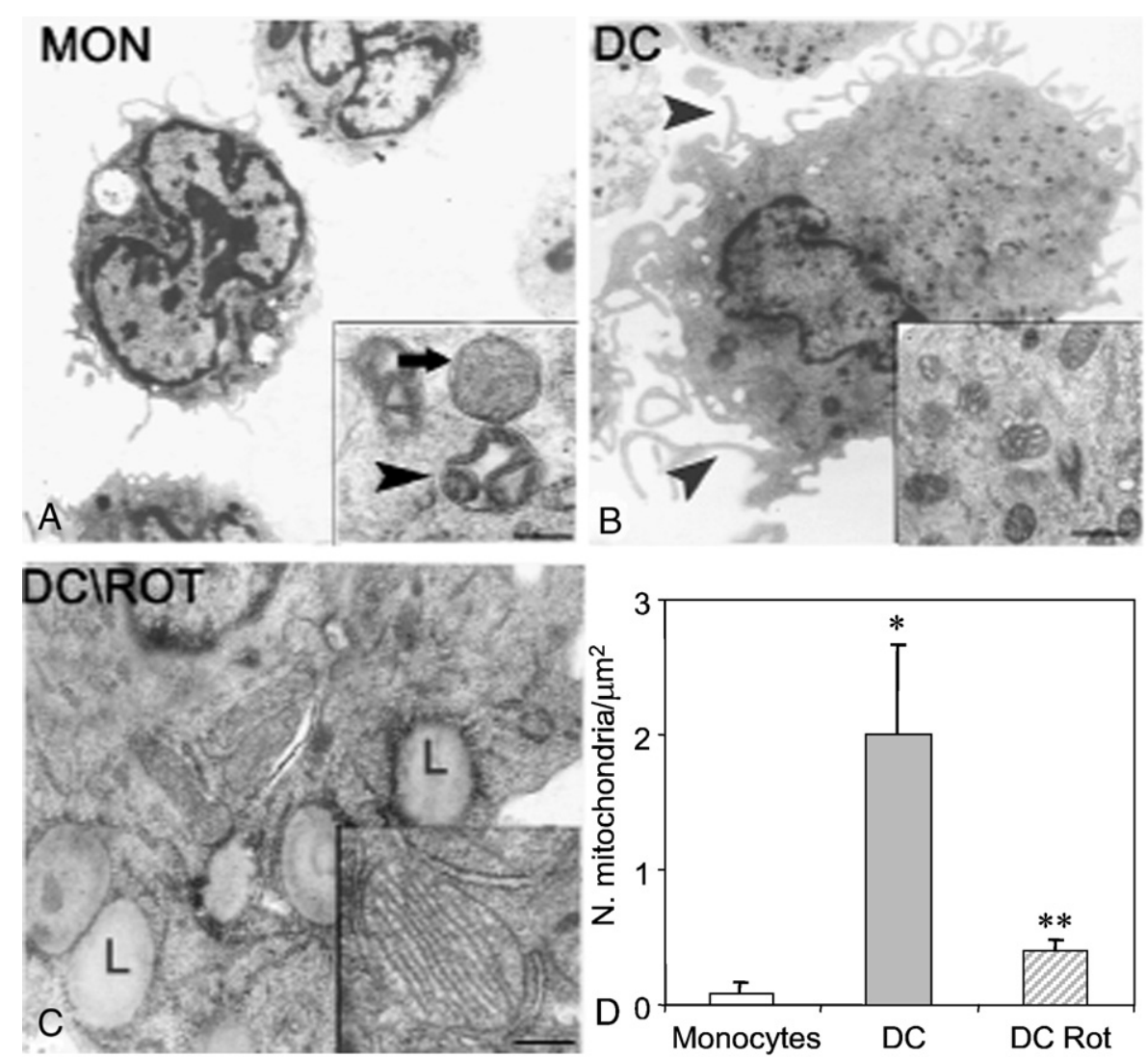

Fig. 2. Transmission electron microscopic examination. (A) Monocytes. The inset shows mitochondria exhibiting orthodox (arrow) and condensed (arrowhead) conformation. (B) Dendritic cell lined at the plasma membrane with fillipodes and pseudopodes (arrowhead) and showing the cytoplasm with numerous condensed mitochondria (inset). (C) Rotenone (Rot)-treated DC showing cytoplasm with lipid droplets (L) and mitochondria with orthodox conformation (inset). Scale bars (original magnification): (A) $1.13 \mu \mathrm{m}$ (inset, $0.25 \mu \mathrm{m}$ ); (B) $1.13 \mu \mathrm{m}$ (inset, $0.41 \mu \mathrm{m}$ ); (C) $0.35 \mu \mathrm{m}$ (inset, $0.24 \mu \mathrm{m}$ ). (D) Statistical analysis of the number of mitochondria/ $\mu \mathrm{m}^{2}$ in monocytes, DC, and rotenone-treated DC. Mean values \pm SEM are indicated $(* p<0.05$ DC vs monocytes; $* * p<0.01$ DC Rot vs DC). 
A

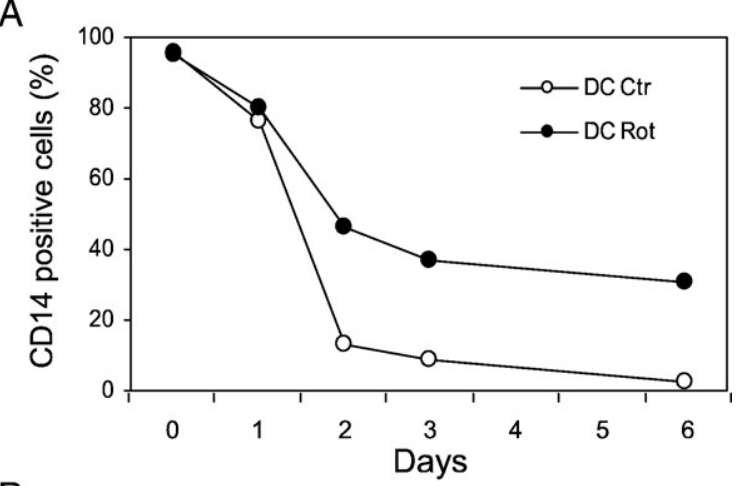

B

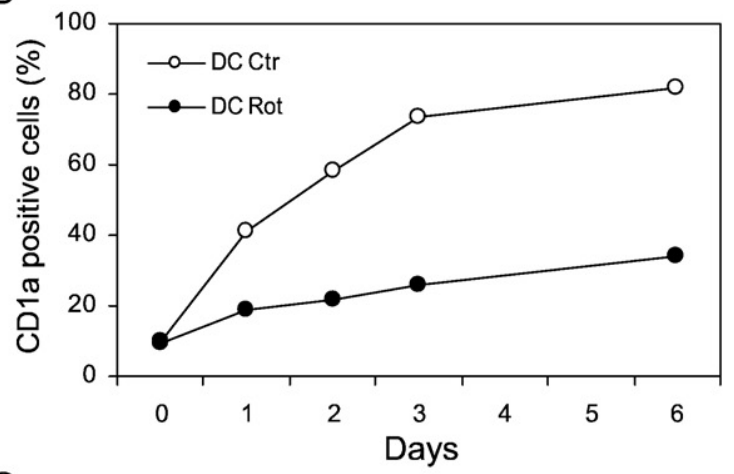

C

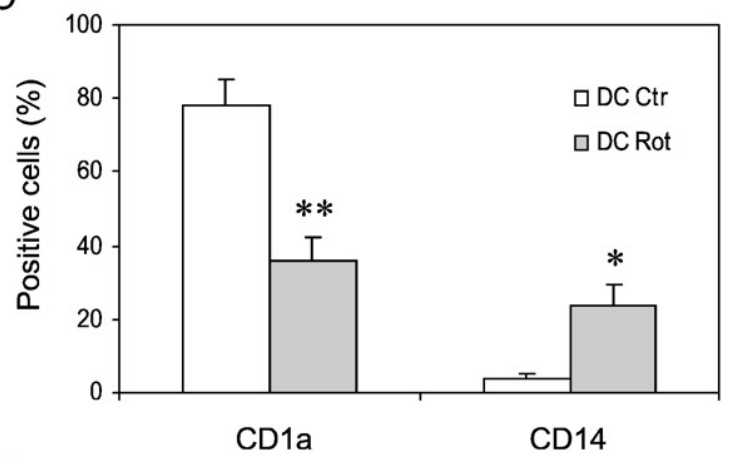

D

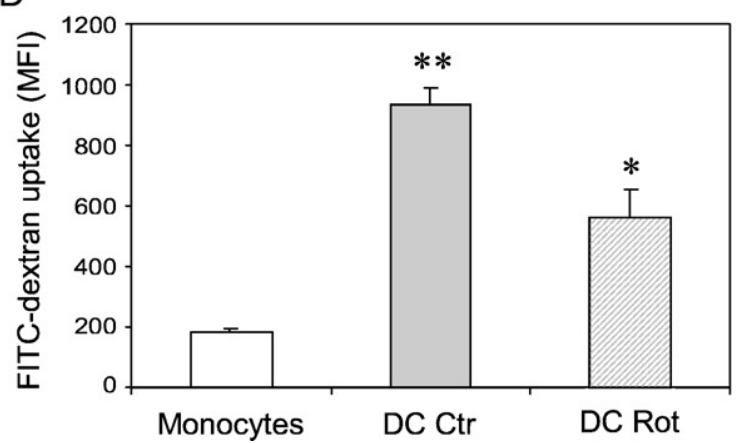

Fig. 3. Phenotypical and functional analysis of DC: effect of rotenone. (A) CD14 and (B) CD1a expression during the differentiation process. Values are expressed as percentage of positive cells. (C) Statistical analysis from five independent experiments. Mean values \pm SEM are indicated $(* p<0.05$ and $* * p<0.01$ DC Rot vs DC Ctr). (D) FITC-dextran uptake, expressed as mean fluorescence intensity (MFI), in monocytes, DC, and rotenone-treated DC. Values are means \pm SEM from at least three experiments $\left({ }^{*} p<0.05\right.$ DC Rot vs DC Ctr; ${ }^{* *} p<0.01$ DC Ctr vs monocytes).
The endocytic activity of DC, measured by monitoring the uptake of FITC-dextran, is shown in Fig. 3D. After the differentiation, immature DC showed a fivefold increased endocytic activity compared to monocytes. However, in rotenone-treated DC the FITC-dextran uptake was largely decreased. All the above results indicate that the addition of rotenone impairs the differentiation process of monocytes into DC.

Fig. 4 shows the effects of rotenone on cellular ATP content and lactate production during DC differentiation. Rotenone treatment, although ineffective at day +1 , resulted subsequently in a reduction in the ATP content increase induced by the differentiation process (Fig. 4A). This effect was accompanied by a progressively increased release of lactate into the culture medium (Fig. 4B), indicating a change in the metabolic and energetic state of these cells.

\section{Analysis of GM-CSF-induced ROS production}

GM-CSF has been reported to cause a rapid increase in ROS production in several cell lines $[11,27,28]$. Thus, experiments were carried out to ascertain whether GM-CSF increased ROS generation in monocytes. ROS production was measured in monocytes cultured either in the absence or in the presence of rotenone (Fig. 5). The maximal ROS production, revealed by DCF fluorescence, was observed early within 10-30 min after the addition of growth factors and consisted in around a twofold increase compared to the basal level, then a leaning plateau was reached (Fig. 5, left). The presence of rotenone in the monocyte culture induced a significant decrease in ROS levels (43.2\% inhibition) (Fig. 5, right). Moreover, when a classical antioxidant such as catalase was used instead of rotenone, a similar drop in ROS production was observed. Catalase treatment also caused a significant reduction in CD1a expression $(42 \pm 8.2 \%, n=3$, positive cells at day +6$)$, comparable to that observed in rotenone-treated cells (see Fig. 3). Furthermore, the respiratory activity measured at day +6 in catalase-treated cells was lower than that exhibited by control DC $\left(1.7 \pm 0.2\right.$ vs $3.4 \pm 0.5 \mathrm{nmol} \mathrm{O}_{2} / \mathrm{min} / 10^{6}$ cells $)$. Importantly, catalase did not affect the respiratory activity when added to precursor monocytes. These results suggest that the differentiation process of DC can be regulated through the action of intracellular redox state induced by growth factors on monocytes.

Laser scanning confocal microscopy analysis showed that DCF fluorescence colocalized with MitoTracker red, a mitochondrial-specific probe, consistent with a prevalent contribution of the organelle to ROS formation (Fig. 6A). Rotenone treatment resulted in a decrease in DCF fluorescence, whereas it was ineffective on MitoTracker red fluorescence. Fluorescence intensity profile analysis (Fig. 6B) showed that rotenone decreased the green fluorescence by around 50\% (cf. Fig. 5).

\section{Discussion}

We have characterized here mitochondrial functions of human monocytes and monocyte-derived DC. DC exhibit a much higher endogenous respiratory activity compared to monocytes and a substantially higher respiratory control ratio (Fig. 1). This finding, together with the observation that DC seemed to respire 
A
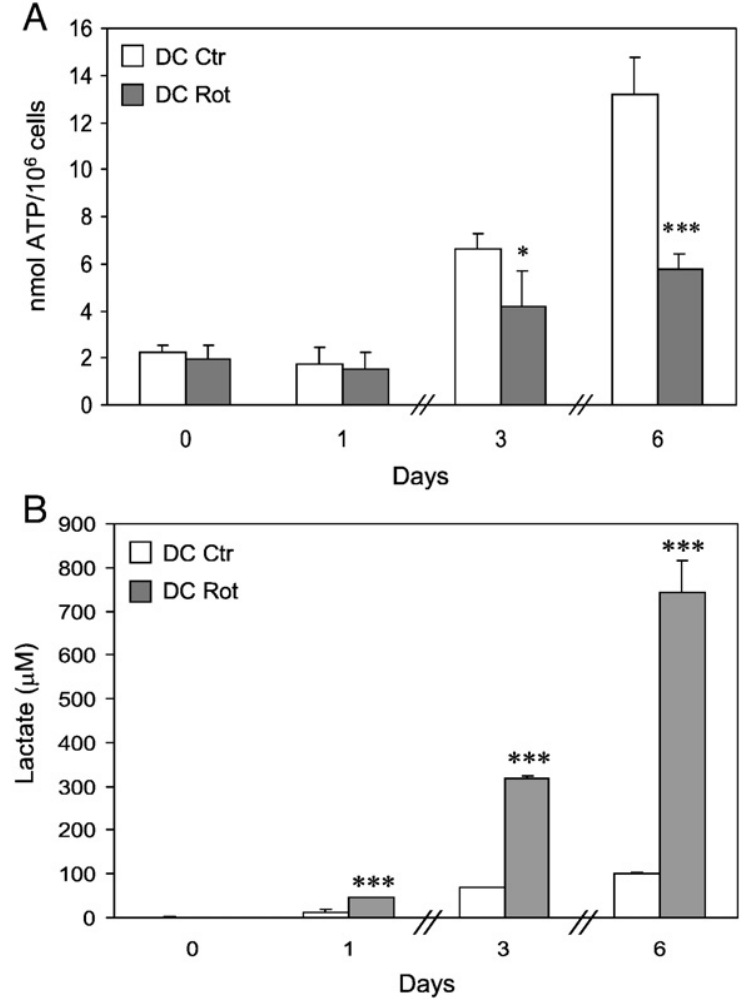

Fig. 4. Effects of rotenone on intracellular ATP content and lactate production during the differentiation process. (A) ATP levels, expressed as nmol ATP $/ 10^{6}$ cells, were measured from day 0 , in monocytes, up to day +6 , in DC differentiated in the absence or presence of rotenone. The means $\pm \mathrm{SEM}$ of at least three independent experiments are indicated $\left({ }^{*} p<0.05\right.$ and ${ }^{* * *} p<0.001$ DC Rot vs DC Ctr). (B) Lactate released in the culture medium was assayed as described under Materials and methods. Values are expressed as $\mu \mathrm{M}$ lactate released and are the means \pm SEM from three different experiments $(* * * p<0.001$ DC Rot vs DC Ctr).

prevalently under State 3, at least under test conditions, would indicate a high capacity of these cells to synthesize ATP. This suggestion is substantiated by direct measurements of ATP production during the differentiation process. After a lag of 1 day,
ATP production increased following almost a saturation curve. The activity of citrate synthase, which is a matrix marker enzyme, nearly paralleled the increase in ATP production. It is conceivable that the dynamic changes in energy metabolism would prepare DC to support energy-dependent processes such as endocytic activity, which greatly increases during the differentiation process (Fig. 3D). Increased ATP production would also be required to support DC migration. This role for mitochondrial ATP has recently been found in T cells, where mitochondrial redistribution occurs in the cytoplasm to regulate the motor of these migratory cells [27]. Whether mitochondria redistribute during DC migration has to be still ascertained.

TEM analysis revealed that an active mitochondrial biogenesis took place during the differentiation and may account for the increased respiratory and citrate synthase activities, as well as ATP production. These morphological and functional changes are triggered by the addition of GM-CSF and interleukin-4 to the culture medium of monocytes. GM-CSF is known to induce a rapid increase in ROS for signaling $[11,28,29]$. Under our experimental conditions a peak of ROS generation was observed within 10-30 min after the addition of GM-CSF to monocytes, whereas ATP production and citrate synthase activity start to increase at least 1 day after. Thus the increased ROS generation seems to precede the readjustment of energy metabolism and to be a cause rather than a consequence of the increased mitochondrial mass accompanying cell differentiation.

The origin of ROS associated with the action of growth factors is widely investigated. We have used in this work rotenone, the inhibitor of the respiratory chain Complex I, to modulate mitochondrial basal ROS generation. This approach, which has been pursued in many laboratories, has produced conflicting results reporting that rotenone could elevate cellular ROS generation in some cases [16,30,31], but reduce ROS production in others $[12,15,32-34]$. Data have recently been produced showing that ROS generated during the oxidation of NAD-dependent substrates are greatly enhanced by rotenone and exclusively released into the mitochondrial matrix [15,16], where they are dissipated by the matrix antioxidant defense. Mn-SOD will form

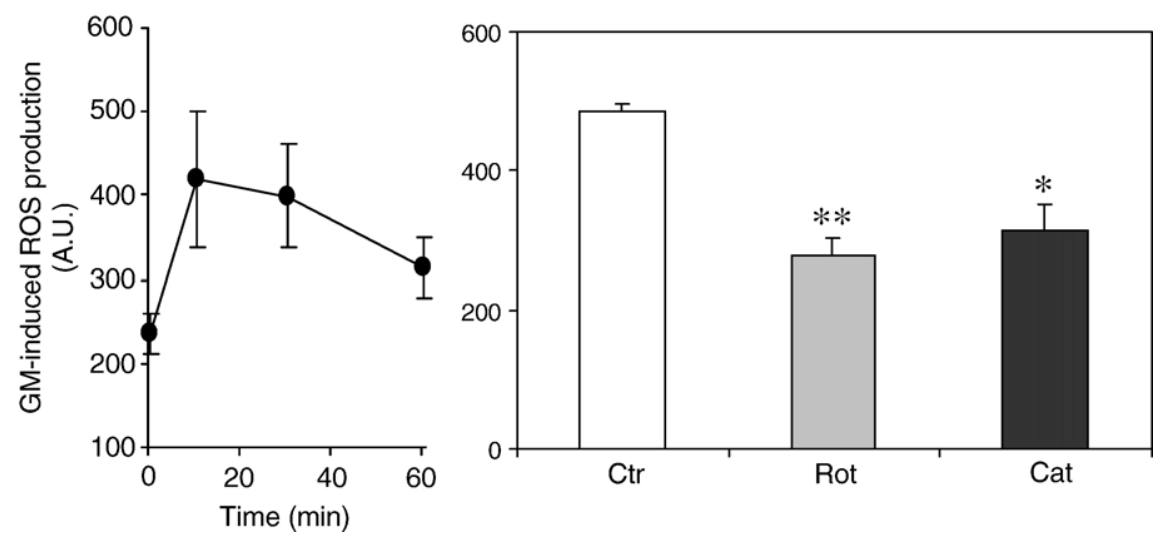

Fig. 5. GM-CSF-induced ROS generation in monocytes: effects of rotenone and catalase. Fluorimetric determination of ROS production was carried out as described under Materials and methods. Left: Time course of ROS production in monocytes initiated by the addition of GM-CSF and IL-4. Right: Effects of rotenone and catalase treatment on ROS production. Values reported (arbitrary units, means \pm SEM from four separate experiments) refer to ROS detected 10-30 min after GM-CSF addition $\left({ }^{*} p<0.05\right.$ catalase-treated vs control monocytes; ${ }^{*} p<0.01$ rotenone-treated vs control monocytes). 
A
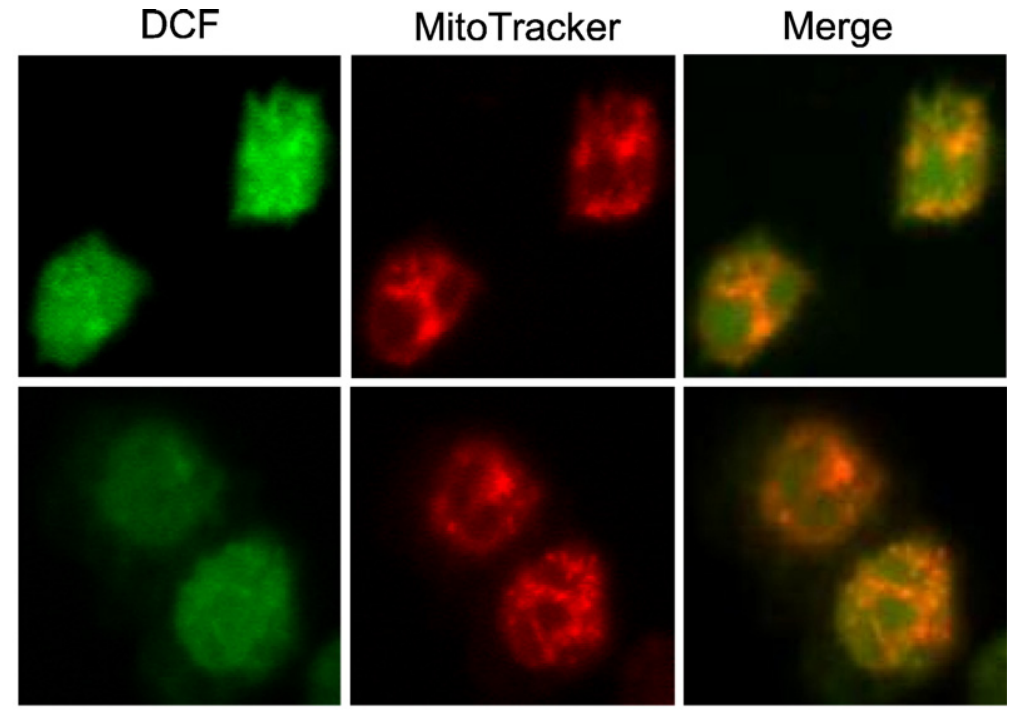

$\mathrm{B}$

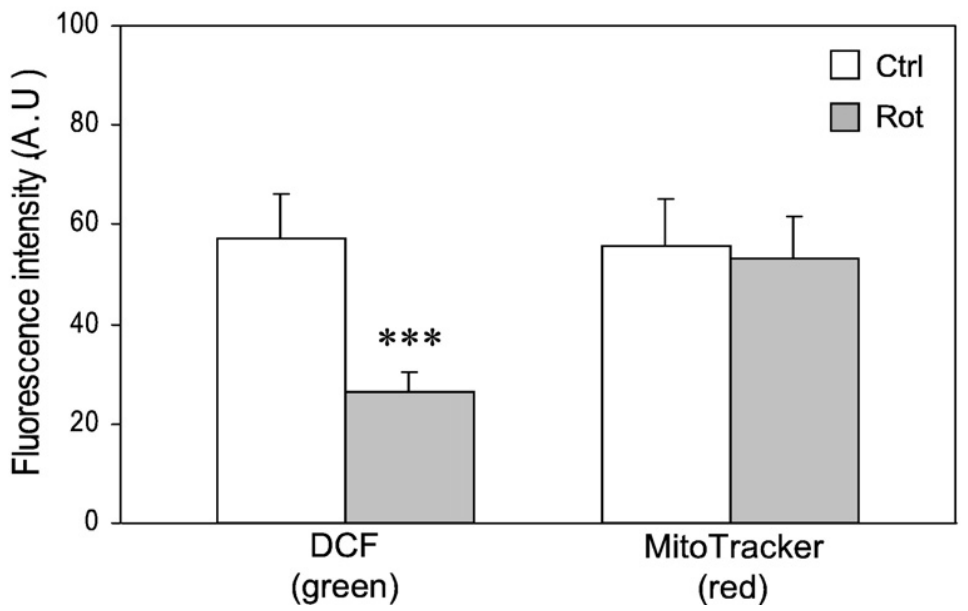

Fig. 6. Confocal microscopy analysis of monocytes. (A) Control and rotenone-treated GM-CSF-stimulated monocytes were incubated for 30 min in the presence of the indicated dyes and analyzed by confocal microscopy (for details see Materials and methods and the legend to Fig. 5). The images are representative of three different experiments. (B) Quantitative analysis of green (DCF) and red (MitoTracker) fluorescence signals was carried out on 10 randomly selected single cells from different microscopic fields for each experiment. The values of fluorescence intensity (arbitrary units) are means $\pm \mathrm{SEM}(* * * p<0.001 \mathrm{Rot} v \mathrm{Ctrl}$ ).

$\mathrm{H}_{2} \mathrm{O}_{2}$ from superoxide, with $\mathrm{H}_{2} \mathrm{O}_{2}$ in turn inactivated by glutathione peroxidase or catalase when present $[35,36]$. In contrast, electron transport within Complex III causes an antimycin-promoted production of ROS which are released on both sides of the inner membrane $[15,16]$. The hydrogen peroxide now generated at the cytosolic side of the inner membrane leaks out from the mitochondria and can be detected by suitable measuring systems. Thus, according to this model, rotenone, while inducing a substantial increase in matrix hydrogen peroxide from Complex I-generated superoxide anion, would cause a decrease in cytosolic hydrogen peroxide deriving from Complex III, just by limiting electron flow into this complex. It is then possible that rotenone treatment of cells or isolated mitochondria oxidizing NAD-linked substrates can result in either a decrease or an increase in extramitochondrial hydrogen peroxide generation, depending on the cell type involved and, in particular, on the activity of matrix antioxidant systems. This point needs, however, further investigation to be better defined.
We have shown here that rotenone caused a significant decrease in ROS production by mitochondria in GM-CSF-stimulated monocytes (Figs. 5 and 6) and this was accompanied by impairment of their differentiation as revealed by the inhibition of the endocytic activity (Fig. 3D), reduction in the dynamic changes of the mitochondrial energy metabolism (Fig. 4), as well as low levels of the marker CD1a (Figs. 3B and C). The latter effects were also elicited by catalase treatment, which caused a comparable drop in ROS generation (Fig. 5). These results indicate that ROS, as demonstrated in the activation and maturation processes [22-24], also play a crucial role in DC differentiation and that mitochondria are an important source of ROS in the process.

The question may then arise whether rotenone inhibits DC differentiation by an indirect mechanism, for example, by causing energy failure. Although such effect cannot be excluded along the various differentiation steps, the above hypothesis is unlike because (i) the presence of rotenone did not impair cell viability and rotenone-treated cells exhibit mitochondria in the 
orthodox conformation, as revealed by TEM analysis (Fig. 2C); (ii) 1 day after the addition of rotenone, ATP production, mostly supported by anaerobic glycolysis (Fig. 4B), was almost similar to that found in control DC and was continuously increasing in the remaining days, although to a lesser extent with respect to control DC (Fig. 4A); and (iii) the rotenone effect was reproduced by catalase, which did not affect the oxidative phosphorylation.

As it has been widely suggested, ROS may act as a second messenger by a mechanism including modulation of the thiol proteome. This in turn would control the activity of protein kinases and phosphatases. Sattler et al. did in fact show that rotenone-sensitive mitochondrial ROS as well as exogenously given hydrogen peroxide induced an increase in tyrosine phosphorylation of cellular proteins and inhibition of protein phosphatases in hematopoietic cells [12]. Furthermore Watanabe et al. showed that mitochondrially generated hydrogen peroxide led to the activation of extracellular signal-regulated kinase in endothelial cells [31].

It is worthwhile considering, however, that rotenone addition to cell culture causes, on the other hand, a substantial increase in cellular NADH, because under these conditions cells are dependent on glycolysis for ATP generation (see Fig. 4). Thus the possibility that the DC differentiation process may depend on the reduction level of the $\mathrm{NAD}^{+} / \mathrm{NADH}$ couple also has to be considered. Such a role for NADH has been recently suggested by Pelicano et al. [37], who found that in rotenone-treated parental Raji cancer cells as well as in derived clones of respirationdeficient cells $\left(\rho^{-}\right)$, the increase in NADH level inactivated the phosphatase PTEN through a redox-mediated mechanism. Thus, it can be hypothesized that the cellular redox tone may regulate the sensitivity of redox signaling pathways to ROS in the DC differentiation process.

\section{Acknowledgments}

This work was financially supported by a grant from the National Research Project (PRIN) on "Bioenergetics: functional genomics, functional mechanisms, and physiopathological aspects." We thank Professor Sergio Papa for suggestions and critical reading of the manuscript. The help of Dr. Michele Scivetti in confocal microscopy analysis, of Dr. Emanuela Blanco in chemiluminescence experiments, of Dr. Elisabetta Crollo of the local blood bank and of Dr. Rossella Crollo for technical assistance in FACS analysis is also acknowledged.

\section{References}

[1] Banchereau, J.; Steinman, R. M. Dendritic cells and the control of immunity. Nature 392:245-252; 1998.

[2] Steinman, R. M.; Hawiger, D.; Nussenzweig, M. C. Tolerogenic dendritic cells. Annu. Rev. Immunol. 21:685-711; 2003.

[3] Sallusto, F.; Mackay, C. R.; Lanzavecchia, A. The role of chemokine receptors in primary, effector, and memory immune responses. Annu. Rev. Immunol. 18:593-620; 2000.

[4] Del Prete, A.; Locati, M.; Otero, K.; Riboldi, E.; Mantovani, A.; Vecchi, A.; Sozzani, S. Migration of dendritic cells across blood and lymphatic endothelial barriers. Thromb. Haemost. 95:22-28; 2006.
[5] Banchereau, J.; Briere, F.; Caux, C.; Davoust, C.; Lebecque, S.; Liu, J. Y.; Pulendran, B.; Palucka, K. Immunobiology of dendritic cells. Annu. Rev. Immunol. 18:767-811; 2000.

[6] Romani, N.; Gruner, S.; Brang, D.; Kampgen, E.; Lenz, A.; Trockenbacher, B.; Konwalinka, G.; Fritsch, P. O.; Steinman, R. M.; Schuler, G. Proliferating dendritic cell progenitors in human blood. J. Exp. Med. 180: 83-93; 1994.

[7] Sallusto, F.; Lanzavecchia, A. Efficient presentation of soluble antigen by cultured human dendritic cells is maintained by granulocyte/macrophage colony-stimulating factor plus interleukin 4 and downregulated by tumor necrosis factor alpha. J. Exp. Med. 179:1109-1118; 1994.

[8] Reth, M. Hydrogen peroxide as second messenger in lymphocyte activation. Nat. Immunol. 3:1129-1134; 2002

[9] Forman, H. J.; Torres, M.; Fukuto, J. Redox signaling. Mol. Cell. Biochem. 234-235:49-62; 2002.

[10] Sauer, H.; Wartenberg, M.; Hescheler, V. Reactive oxygen species as intracellular messengers during cell growth and differentiation. Cell. Physiol. Biochem. 11:173-186; 2001.

[11] Sattler, M.; Winkler, T.; Verma, S.; Byrne, C. H.; Shrikhande, G.; Salgia R.; Griffin, J. D. Hematopoietic growth factors signal through the formation of reactive oxygen species. Blood 93:2928-2935; 1999.

[12] Sattler, M.; Verma, S.; Shrikhande, G.; Byrne, C. H.; Pride, Y. B.; Winkler, T.; Greenfield, E. A.; Salgia, R.; Griffin, J. D. The BCR/ABL tyrosine kinase induces production of reactive oxygen species in hematopoietic cells. J. Biol. Chem. 275:24273-24278; 2000.

[13] Thannickal, V. J.; Fanburg, B. L. Reactive oxygen species in cell signaling. Am. J. Physiol. Lung Cell. Mol. Physiol. 279:L1005-L1028; 2000.

[14] Cadenas, E. Mitochondrial free radical production and cell signaling. Mol. Aspects Med. 25:17-26; 2004.

[15] Chen, Q.; Vazquez, E. J.; Moghaddas, S.; Hoppel, C. L.; Lesnefsky, E. J. Production of reactive oxygen species by mitochondria: central role of complex III. J. Biol. Chem. 278:36027-36031; 2003.

[16] Muller, F. L.; Liu, Y.; Van Remmen, H. Complex III releases superoxide to both sides of the inner mitochondrial membrane. J. Biol. Chem. 279: 49064-49073; 2004.

[17] Savina, A.; Jancic, C.; Hugues, S.; Guermonprez, P.; Vargas, P.; Moura I. C. A.; Lennon-Dumenil, M.; Seabra, M. C.; Raposo, V.; Amigorena, S. NOX2 controls phagosomal $\mathrm{pH}$ to regulate antigen processing during crosspresentation by dendritic cells. Cell 126:205-218; 2006.

[18] Vignais, P. V. The superoxide-generating NADPH oxidase: structural aspects and activation mechanism. Cell. Mol. Life Sci. 59:1428-1459; 2002.

[19] Babior, B. M.; Lambeth, J. D.; Nauseef, W. The neutrophil NADPH oxidase. Arch. Biochem. Biophys. 397:342-344; 2002.

[20] Vulcano, M.; Dusi, S.; Lissandrini, D.; Badolato, R.; Mazzi, P.; Riboldi, E.; Borroni, E.; Calleri, A.; Donini, M.; Plebani, A.; Notarangelo, V.; Musso, T.; Sozzani, S. Toll receptor-mediated regulation of NADPH oxidase in human dendritic cells. J. Immunol. 173:5749-5756; 2004.

[21] Donini, M.; Zenaro, E.; Tamassia, N.; Dusi, S. NADPH oxidase of human dendritic cells: role in Candida albicans killing and regulation by interferons, dectin-1 and CD206. Eur. J. Immunol. 37:1194-1203; 2007.

[22] Rutault, K.; Alderman, C.; Chain, B. M.; Katz, D. R. Reactive oxygen species activate human peripheral blood dendritic cells. Free Radic. Biol. Med. 26:232-238; 1999.

[23] Kantengwa, S.; Jornot, L.; Devenoges, C.; Nicod, L. P. Superoxide anions induce the maturation of human dendritic cells. Am. J. Respir. Crit. Care Med. 167:431-437; 2003.

[24] Matsue, H.; Edelbaum, D.; Shalhevet, D.; Mizumoto, N.; Yang, C.; Mummert, M. E.; Oeda, J.; Masayasu, H.; Takashima, A. Generation and function of reactive oxygen species in dendritic cells during antigen presentation. J. Immunol. 171:3010-3018; 2003.

[25] Brandt, R. B. Determination of D-lactate in plasma. Methods Enzymol. 89 (Pt D):35-40; 1982.

[26] Tarpey, M. M.; Wink, D. A.; Grisham, M. B. Methods for detection of reactive metabolites of oxygen and nitrogen: in vitro and in vivo considerations. Am. J. Physiol., Regul. Integr. Comp. Physiol. 286:R431-R444; 2004 
[27] Campello, S.; Lacalle, R. A.; Bettella, M.; Mañes, S.; Scorrano, L.; Viola, A. Orchestration of lymphocyte chemotaxis by mitochondrial dynamics. J. Exp. Med. 203:2879-2886; 2006.

[28] Sinha, A.; Singh, A.; Satchidanandam, V.; Natarajan, K. Impaired generation of reactive oxygen species during differentiation of dendritic cells (DCs) by Mycobacterium tuberculosis secretory antigen (MTSA) and subsequent activation of MTSA-DCs by mycobacteria results in increased intracellular survival. J. Immunol. 177:468-478; 2006.

[29] Carcamo, J. M.; Borquez-Ojeda, O.; Golde, D. W. Vitamin C inhibits granulocyte macrophage-colony-stimulating factor-induced signaling pathways. Blood 99:3205-3212; 2002.

[30] Li, N.; Ragheb, K.; Lawler, G.; Sturgis, J.; Rajwa, B.; Melendez, J. A.; Robinson, J. P. Mitochondrial complex I inhibitor rotenone induces apoptosis through enhancing mitochondrial reactive oxygen species production. J. Biol. Chem. 278:8516-8525; 2003.

[31] Watanabe, N.; Zmijewski, J. W.; Takabe, W.; Umezu-Goto, M.; Le Goffe, C.; Sekine, A.; Landar, A.; Watanabe, A.; Aoki, J.; Arai, H.; Kodama, T.; Murphy, M. P.; Kalyanaraman, R.; Darley-Usmar, V. M.; Noguchi, N. Activation of mitogen-activated protein kinases by lysophosphatidylcholine-induced mitochondrial reactive oxygen species generation in endothelial cells. Am. J. Pathol. 168:1737-1748; 2006.
[32] Lesnefsky, E. J.; Chen, Q.; Moghaddas, S.; Hassan, M. O.; Tandler, B.; Hoppel, C. L. Blockade of electron transport during ischemia protects cardiac mitochondria. J. Biol. Chem. 279:47961-47967; 2004.

[33] Li, Y.; Trush, M. A. Diphenyleneiodonium, an NAD(P)H oxidase inhibitor, also potently inhibits mitochondrial reactive oxygen species production. Biochem. Biophys. Res. Commun. 253:295-299; 1998.

[34] Vrablic, A. S.; Albright, C. D.; Craciunescu, C. N.; Salganik, R. I.; Zeisel, S. H. Altered mitochondrial function and overgeneration of reactive oxygen species precede the induction of apoptosis by 1-O-octadecyl-2-methylrac-glycero-3-phosphocholine in p53-defective hepatocytes. FASEB J. 15: 1739-1744; 2001.

[35] Radi, R.; Turrens, J. F.; Chang, L. Y.; Bush, K. M.; Crapo, J. D.; Freeman, B. A. Detection of catalase in rat heart mitochondria. J. Biol. Chem. 266: 22028-22034; 1991.

[36] Salvi, M.; Battaglia, V.; Brunati, A. M.; La Rocca, N.; Tibaldi, E.; Pietrangeli, P.; Marcocci, L.; Mondovi, V.; Rossi, C. A.; Toninello, A. Catalase takes part in rat liver mitochondria oxidative stress defense. J. Biol. Chem. 282:24407-24415; 2007.

[37] Pelicano, H.; Xu, R.H.; Du, M.; Feng, L.; Sasaki, R.; Carew, J.S.; Hu, Y.; Ramdas, L.; Hu, L.; Keating, M.J.; Zhang, W.; Plunkett, W.; Huang. P. Mitochondrial respiration defects in cancer cells cause activation of Akt survival pathway through a redox-mediated mechanism. J. Cell Biol. 175:913-923. 